\title{
Performances économiques et financières de la fertilisation en riziculture pluviale stricte dans la zone sud soudanienne du Burkina Faso
}

\author{
Abdramane SANON ${ }^{1,2 *}$, Alain P.K. GOMGNIMBOU ${ }^{2}$, Hamadé SIGUE ${ }^{3}$, Kalifa \\ COULIBALY $^{1}$, Cheick A. BAMBARA ${ }^{2}$, Willifried SANOU ${ }^{2}$, Sékou FOFANA ${ }^{1,2}$ et \\ Hassan B. NACRO ${ }^{1}$
}

\author{
${ }^{l}$ Laboratoire d'Etude et de Recherche sur la Fertilité du Sol, Institut du Développement Rural, Université Nazi \\ Boni. BP 1091, Bobo-Dioulasso, Burkina Faso. \\ ${ }^{2}$ Laboratoire Sol-Eau-Plante, Programme de Recherche Gestion des Ressources Naturelles et Système de \\ Production, Institut de l'Environnement et de Recherches Agricoles, Station de Farako-Bâ. 01 BP 910 Bobo \\ Bobo-Dioulasso 01, Burkina Faso. \\ ${ }^{3}$ Programme de Recherche Gestion des Ressources Naturelles et Système de Production, Institut de \\ l'Environnement et de Recherches Agricoles, Station de Saria, Koudougou, 04BP8645 Ouagadougou, \\ Burkina Faso. \\ *Auteur correspondant ; E-mail : sdumba8006@gmail.com
}

\author{
Received: 12-05-2021 \\ Accepted: 20-08-2021 \\ Published: 31-08-2021
}

\section{RESUME}

La culture du riz joue un rôle décisif dans les moyens d'existence des producteurs. La production rizicole rencontre des difficultés liées aux coûts de fertilisation. L'étude avait pour objectif d'évaluer les performances économiques et financières des biodéchets et des fertilisants inorganiques en riziculture pluviale stricte. L'évaluation est faite suivante deux démarches : la première consiste à calculer et à comparer les indicateurs de performance, tandis que la deuxième repose sur une matrice de corrélation afin d'identifier les relations entre les indicateurs de performances économiques et financières et rendements de riz paddy. La performance agronomique des options de fertilisation a été évaluée par les rendements du riz paddy récolté dans un essai. A cet effet, un dispositif expérimental en Blocs de Fisher complètement randomisés avec quatre répétitions et dix traitements composites a été installé à la station de recherches de Farako-Ba au Burkina Faso, durant quatre campagnes agricoles de 2016 à 2019. Pour le calcul des indicateurs de performances économiques et financières, l'achat des engrais minéraux et des biodéchets, le coût de la main-d'œuvre pour collecter, transporter et incorporer les fertilisants, le prix moyen d'un kilogramme de riz paddy dans les différents marchés de la zone d'étude ont été prises en considération. Les résultats ont montré que les traitements Fumier de Poule+Urée, Fumier de Poule+Burkina Phosphate+Urée et Compost+Urée présentaient significativement les meilleures performances économiques du point de vue de la marge nette et de la productivité moyenne du travail. Les meilleurs taux de rentabilité interne et du ratio bénéfice sur coût sont enrégistrés par les traitements Fumier de Poule+Urée et Compost+Urée. Il ressort de l'analyse statistique des corrélations positives et significatives entre le rendement du riz paddy et les marges nettes d'une part et d'autre part entre le rendement du riz paddy et la productivité moyenne du travail. Dans un contexte de coûts élevés de la fertilisation, ces résultats révèlent l'intérêt de la combinaison de biodéchets (fumier de poule) avec les engrais minéraux en culture de riz pluvial strict pour une vulgarisation en milieu paysan.

(C) 2021 International Formulae Group. All rights reserved.

Mots clés: riz pluvial strict, fertilisation, performance, productivité. 


\title{
Economic and financial performance of upland rice fertilization in south Sudan zone of Burkina Faso
}

\begin{abstract}
Rice cultivation plays a decisive role in the livelihoods of producers. Rice production encounters difficulties linked to fertilization costs. The study aimed to assess the economic and financial performance of bio-waste and inorganic fertilizers in upland rice cultivation. The assessment is made following two approaches: the first consists in calculating and comparing performance indicators, while the second relies on a correlation matrix in order to identify the relationships between economic and financial performance and returns. To this end, a completely randomized Fisher Block experimental device with four repetitions and ten composite treatments was installed at the Farako-Ba research station in Burkina Faso, during four agricultural campaigns from 2016 to 2019. For the calculation of the indicators economic and financial performance, the purchase of mineral fertilizers and bio-waste, the cost of labor to collect, transport and incorporate fertilizers, the average price of a kilogram of paddy rice in the various markets of the study area have been taken into account. The results showed that the Chicken Manure + Urea, Chicken Manure + Burkina Phosphate + Urea and Compost + Urea treatments significantly presented the best economic performance in terms of net margin and average labor productivity. The best internal rates of return and the benefit-cost ratio are recorded by the Chicken Manure+Urea and Compost+Urea treatments. Statistical analysis shows positive and significant correlations between paddy rice yield and net margins on the one hand and between paddy rice yield and average labor productivity on the other hand. In a context of high fertilization costs, these results reveal the value of the combination of bio-waste (Chicken Manure) with mineral fertilizers in upland rice cultivation for extension in rural areas.

(C) 2021 International Formulae Group. All rights reserved.
\end{abstract}

Keywords: Upland rice; fertilization; performance, productivity.

\section{INTRODUCTION}

Le Burkina Faso a une population estimée à 20487979 habitants dont 73,7\% vivent en milieu rural (INSD, 2020). La population agricole est estimée à 17260000 habitants (MAAH, 2020). Parmi les principales cultures céréalières, le riz occupe une place prépondérante après le mil, le maïs, et le sorgho. La production du riz a été évaluée à 356 280 tonnes en 2018 (MAAH, 2018), mais avec un déficit estimé à 393816 tonnes. Les cinq dernières années, la production nationale de riz ne couvre que $30 \%$ des besoins de consommation de la population (SNDR, 2020). La demande de consommation est en constante augmentation avec un taux d'accroissement de $11 \%$ par an et les besoins annuels de consommation du riz pourraient atteindre 1500000 tonnes d'ici à 2025 (SNDR, 2020). Pour combler le déficit de besoin en consommation, le Burkina Faso a recours à des importations massives. Ainsi, le riz reste la principale charge d'importation de céréales au
Burkina Faso avec une dépense de 69 252,6 millions de FCFA en 2019 (MCIA, 2020).

Il existe trois écologies de systèmes de production rizicole dans le pays : riziculture pluviale stricte, riziculture pluviale de basfonds et riziculture irriguée. Les marges bénéficiaires dégagées par les producteurs après déduction des coûts de production, sont de l'ordre de $60000 \mathrm{FCFA} / \mathrm{Ha}$ en riziculture pluviale stricte, 82000 à 125000 FCFA/ ha en riziculture de bas-fonds, et 168000 à 270000 FCFA/Ha en riziculture irriguée (Eureka, 2005). Cette situation pourrait être liée à la pauvreté générale des terres (DDEA, 2012), au faible niveau de performances techniques et économiques des exploitations familiales. Il faut noter que les options de fertilisation ont été développées pour rendre performants les exploitations rizicoles. En effet, les travaux de Useni et al. (2012), ont montré que la rentabilité optimale est obtenue avec la faible dose de fiente de poulet combinée ou non à la faible dose d'engrais chimiques en production de maïs. D'autres travaux de recherches ont 
montré que, les applications conjointes d'engrais organiques et engrais minéraux entraine des augmentations de la production agricole. Il en résulte aussi, une nette amélioration du capital de fertilité du sol (Ankaza et al., 2003 ; Koné et al., 2011 ; Onaga et al., 2014 ; Adebiyi et al., 2019), l'emploi des fumiers de poule associé avec du Burkina Phosphate et de l'urée est une alternative à la fertilisation du riz pluvial strict (Sanon et al., 2021). L'aspect écologique de ce paquet de fertilisation est bien connu cependant, pas de sa performance économique et financière en condition de riziculture pluviale stricte qui reste ambiguë. Le problème réside entre les traitements appliqués qui pourraient affecter les écarts de rendement (rendement par rapport au potentiel de rendement maximal) dans les deux systèmes (organiques et inorganiques) et la variation de rendement qui peut être observée entre et au sein des systèmes. La compréhension précise de la logique de production peut aider à augmenter la productivité du riz pluvial strict. L'objectif de l'étude était d'évaluer la performance économique et financière des biodéchets et des fertilisants inorganiques en riziculture pluviale stricte. Le présent article porte sur les résultats de l'étude conduite au cours des campagnes agricoles de saison humide 2016-2017-20182019.

\section{MATERIEL ET METHODES}

\section{Site d'étude}

L'étude a été conduite durant quatre campagnes agricoles de 2016 à 2019 à la station de Recherches Environnementales et Agricoles de Farako-Ba. Les coordonnées géographiques du site se situent entre le $11^{\circ} 06$ de latitude Nord et le $4^{\circ} 20$ de longitude Ouest à $405 \mathrm{~m}$ d'altitude. Le climat est de type sud soudanien. Il est caractérisé par la succession de deux principales saisons, la saison sèche (mi-Octobre à Avril) et la saison pluvieuse (Mai à mi-Octobre). La pluviométrie moyenne annuelle en 2019 était de $1371 \mathrm{~mm}$ (MAAH, 2019). Les sols du site d'implantation de l'essai sont des lixisols selon la classification WRB CPCS (Kissou et al., 2014). La production du riz dans la zone est estimée à 30601 tonnes et représentent $8 \%$ de la production nationale en riz (MAAH, 2019).

\section{Matériel végétal et fertilisants utilisés}

La variété de riz FKR59 avec un cycle de 95-100 jours pour un rendement moyen de $5000 \mathrm{~kg} / \mathrm{ha}$ (INERA, 2016) a été utilisée. La bonne réponse à la fertilisation azotée et sa résistance à la pyriculariose expliquent le choix de cette variété. Les fertilisants utilisés sont composés de: Fumiers de Poules avec $\mathrm{N}=$ 3,09\% ; P_total $=2,56$ mg.kg-1 de sol ; K_total $=1,43 ; \mathrm{C} / \mathrm{N}=12,33) ;$ Compost $(\mathrm{N}=2,18 \%$; $\mathrm{P}$ total $=0,026 \mathrm{mg} \cdot \mathrm{kg}^{-1}$ de sol $; \mathrm{K}$ total $=1,8$ mg. $\mathrm{kg}^{-1}$ de sol ; $\left.\mathrm{C} / \mathrm{N}=10,79\right)$; Burkina Phosphate avec: $\mathrm{P}_{2} \mathrm{O}_{5}=25,38 \% ; \mathrm{CaO}=34$, $45 \% ; \mathrm{MgO}=0,27 \%$ Ferral $=6,5 \% ; \mathrm{SiO}_{2}=$ $26,24 \%$;

NPK (14-23-14) et de l'Urée (46\% N).

\section{Dispositif expérimental et conduite de l'essai}

L'essai repose sur un cadre, selon un dispositif en Blocs de Fisher complètement randomisés à 10 traitements (Tableau 2) et 4 répétitions. La superficie totale de l'essai était de $517,5 \mathrm{~m}^{2}(34,5 \mathrm{~m} \mathrm{x} 15 \mathrm{~m})$ et celle des parcelles élémentaires était de $9 \mathrm{~m}^{2}(3 \mathrm{~m} \times 3 \mathrm{~m})$ chacune. Les parcelles élémentaires étaient séparées par des allées de $0,5 \mathrm{~m}$ et les répétitions par des allées de $1 \mathrm{~m}$. La même opération s'est répétée chaque année. L'étude a été réalisée au cours des campagnes agricoles 2016, 2017, 2018 et 2019.

Chaque parcelle élémentaire qui a une superficie de $9 \mathrm{~m}^{2}$, a porté les types de formules de fertilisation correspondantes. La préparation du terrain a consisté au défrichement et au labour à plat. Les pailles de riz, ont été enfouies lors du labour et le hersage est intervenu juste après. Le compost à base de paille de riz et le Burkina Phosphate ont été enfouies 5 jours avant semis. Le fumier de poule a été appliqué par enfouissement en deux fractions : 5000 $\mathrm{kg} / \mathrm{ha}$ à 5 jours avant semis et $2500 \mathrm{~kg} / \mathrm{ha}$ à 45 jours après semis. Le NPK a été appliquée à 14 Jours après semis et l'urée sous forme fractionnée soit $35 \mathrm{~kg} / \mathrm{ha}$ au tallage et $65 \mathrm{~kg} / \mathrm{ha}$ à l'épiaison. Le semis a été réalisé en raison d'une pincée de grains de riz par poquet. 
L'écartement était de $20 \mathrm{~cm}$ entre les poquets et $20 \mathrm{~cm}$ entre les lignes. Le démariage est intervenu 15 jours après semis, à raison de 2 plants par poquet.

\section{Méthodes}

\section{Performance technique}

La performance technique des pratiques culturales de gestion de la fertilité des sols a été évaluée par les rendements paddy du riz récolté. Le rendement paddy (t/ha) a été déterminé en utilisant la formule donnée par Lacharme (2001) :

Rendement Paddy $=$

$\frac{\mathrm{NP}}{h a} X \frac{\mathrm{NT}}{P} X \frac{\mathrm{Npa}}{T} X \frac{\mathrm{NG}}{P a} X N G$

Avec $: \frac{\mathrm{NP}}{h a}=$ nombre de plants/hectare $=\frac{\mathrm{NP}}{m^{2}} \times 10$ 000

$\frac{\mathrm{NT}}{P}=$ nombre de talles/pieds

$\frac{\mathrm{Npa}}{T}=$ nombre de panicule/talle

$\frac{\mathrm{NG}}{P a}=$ nombre de grains/panicules

$\mathrm{PG}=$ poids d'un grain .

\section{Indicateurs de performance économique}

Les performances économiques des traitements (pratiques culturales de gestion de la fertilité des sols) ont été estimées par quatre (04) différents indicateurs que sont : la Marge Nette de production (MN), la Productivité Moyenne du Travail (PML), le Taux de Rentabilité Interne (TRI) et le ratio Bénéfice sur Coût (RBC).

\section{Marge Nette (MN)}

Exprimée en FCFA/ha, la Marge Nette de production est obtenue en déduisant du produit brut en valeur (PBV) à l'hectare, les coûts totaux $(\mathbf{C T})$ à l'hectare ou en déduisant de la marge brute (MB) les coûts fixes $(\mathbf{C F})$ à l'hectare.

La formule proposée par Yegbemey et al. (2014) a été utilisée :

$$
\begin{aligned}
& \mathrm{MN}=\mathrm{PBV}-\mathrm{CT}=\mathrm{PBV}-\mathrm{CV}-\mathrm{CF}=\mathrm{MB}- \\
& \mathrm{CF}
\end{aligned}
$$

CV : les coûts variables, correspondent aux dépenses liées à l'acquisition d'intrants (engrais, insecticides, pesticides et main d'œuvre) (en FCFA/ha) ;

CF : les coûts fixes correspondent aux dépenses faites par l'exploitation mais non liées au volume de production : ce sont principalement les dépenses d'investissement. Les coûts fixes sont déterminés en appliquant un taux d'amortissement linéaire à la valeur totale d'acquisition du matériel. Ce taux correspond à l'inverse de la durée de la vie de ce matériel.

Si MN>0, l'activité de production est économiquement rentable. Le produit brut permet donc de couvrir toutes les charges de production, par contre $\mathbf{s i} \mathbf{M N}<\mathbf{0}$, l'activité n'est pas rentable du point de vue économique.

\section{Productivité Moyenne du Travail (PML)}

Cet indicateur de rentabilité est défini comme la marge nette par unité de maind'œuvre familiale utilisée pour la production. Mathématiquement, elle est exprimée par la formule utilisée par Labiyi et al. (2019):

PML $=\frac{\text { MN }}{\text { MO }}$

Où $\mathbf{M N}$ représente la marge nette de l'activité de production (en FCFA/ha) et MO la quantité totale de main d'œuvre familiale utilisée (HJ/ha). Ce faisant PML est exprimée en FCFA/HJ.

Si PML>p, on peut conclure que l'activité est économiquement rentable du point de vue du salaire obtenu. Si par contre $\mathbf{P M L}<\mathbf{p}$, alors l'activité n'est pas économiquement rentable du point de vue du salaire obtenu. (Avec p, le salaire journalier payé à un homme dans la zone d'étude). A la station de Farako-Bâ, la valeur p est égale à 1147,14 FCFA / HJ.

\section{Taux de Rentabilité Interne (TRI)}

Le Taux de Rentabilité Interne ou TRI est un indicateur d'analyse de la rentabilité financière d'une activité de production.

$$
\text { TRI }=\frac{\text { MN-MOV }}{C T+\text { MOV }}
$$

La même formule a été utilisée par Labiyi et al. (2019).

Avec : MN la marge nette de l'activité de production (en FCFA/ha);

CT les coûts totaux en FCFA/ha qui ne prennent pas en compte la valeur de la main- 
d'œuvre familiale utilisée et MOV la quantité totale de main d'œuvre familiale utilisée (en Homme. Jour/ha). Cette valeur de la maind'œuvre familiale est obtenue en multipliant le prix quotidien $\mathbf{p}$ de la main-d'œuvre dans la zone d'étude par la quantité totale de maind'œuvre utilisée (MO) dans la zone de FarakoBâ $\mathbf{p}=1147,14$ FCFA /HJ

Le TRI mesure la marge nette par unité de capital investi. Dans ce cas, le capital investi correspond aux coûts totaux de production (Sigue et al., 2019). Il permet également d'apprécier la capacité d'une exploitation à rentabiliser le capital. Le TRI est interprété en comparant au taux d'intérêt $\mathbf{i}=24 \%$, appliqué par les institutions bancaires ou de micro finance dans la zone d'étude. Ainsi, si TRI > i, l'activité est économiquement rentable du point de vue de l'investissement. Si TRI $<$ i, l'activité n'est pas rentable du point de vue de l'investissement.

Le Ratio Bénéfice sur Coût ou RBC exprime le gain financier total obtenu par l'investissement d'une unité monétaire (1 franc CFA par exemple). Soit B l'ensemble des bénéfices obtenus après un investissement total C. Il vient alors qu'en économie agricole, $\mathbf{B}$ est désigné par le produit brut obtenu en valeur et C par l'ensemble de tous les coûts exprimés en valeur, y compris la main d'œuvre familiale.
Ainsi, si PBV est le produit brut en valeur, les coûts totaux qui ne prennent pas en compte la valeur de la main-d'œuvre familiale et la valeur totale de la main-d'œuvre familiale utilisée, la formule proposée par Sigue et al. (2019) a été utilisée. Ainsi, on a :

$\mathbf{R B C}=\frac{\mathrm{PBV}}{C T+\mathrm{MOV}}$

MOV : dans la pratique, MOV est obtenue en multipliant la quantité physique de main d'œuvre familiale totale par le prix moyen de la main-d'œuvre salariée dans la zone de l'étude.

\section{Analyse statistique}

L'analyse de variance (ANOVA) et la matrice de corrélation ont été réalisées à l'aide du logiciel GENTSTAT Discovery version 11.1. Le test de Fisher a été utilisé pour la comparaison des moyennes lorsque l'analyse de variance révèle des différences significatives entre les traitements.

Dans le cadre de cette étude, aucun investissement n'a été entrepris nécessitant le calcul des amortissements, alors, les coûts variables et les coûts fixes sont nettement les mêmes et correspondent aux coûts totaux ou coût de Production.

Tableau 1 : Coordonnées de la parcelle d'essai.

\section{Points de la parcelle Coordonnées}

\begin{tabular}{ll}
\hline Point 1 & $11^{\circ} 05.445^{\prime}$ nord, $004^{\circ} 19.674^{\prime}$ ouest \\
Point 2 & $11^{\circ} 05.452^{\prime}$ nord, $004^{\circ} 19.668^{\prime}$ ouest \\
Point 3 & $11^{\circ} 05.466^{\prime}$ nord, $004^{\circ} 19.681^{\prime}$ ouest \\
Point 4 & $11^{\circ} 05.461^{\prime}$ nord, $004^{\circ} 19.687^{\prime}$ ouest
\end{tabular}


Tableau 2 : Liste de traitements appliqués.

\begin{tabular}{cll}
\hline Traitements & Libellé & Compositions \\
\hline T1 & Témoin absolu & Sans apport de fertilisant \\
T2 & NPK+Urée & NPK $(200 \mathrm{~kg} / \mathrm{ha})+$ Urée $(100 \mathrm{~kg} / \mathrm{ha})$ \\
T3 & BP+Urée & BP $(500 \mathrm{~kg} / \mathrm{ha})+$ Urée $(100 \mathrm{~kg} / \mathrm{ha})$ \\
T4 & FP & FP $(7500 \mathrm{~kg} / \mathrm{ha})$ \\
T5 & FP+Urée & FP $(7500 \mathrm{~kg} / \mathrm{ha})+$ Urée $(100 \mathrm{~kg} / \mathrm{ha})$ \\
T6 & FP+BP & FP $(7500 \mathrm{~kg} / \mathrm{ha})+$ BP $(500 \mathrm{~kg} / \mathrm{ha})$ \\
T7 & FP+BP+Urée & FP $(7500 \mathrm{~kg} / \mathrm{ha})+$ BP $(500 \mathrm{~kg} / \mathrm{ha})+$ Urée $(100 \mathrm{~kg} / \mathrm{ha})$ \\
T8 & Compost+Urée & Compost $(5000 \mathrm{~kg} / \mathrm{ha})+$ Urée $(100 \mathrm{~kg} / \mathrm{ha})$ \\
T9 & Compost+BP & Compost $(5000 \mathrm{~kg} / \mathrm{ha})+\mathrm{BP}(500 \mathrm{~kg} / \mathrm{ha})$ \\
T10 & Compost+BP+Urée & Compost $(5000 \mathrm{~kg} / \mathrm{ha})+\mathrm{BP}(500 \mathrm{~kg} / \mathrm{ha})+$ Urée $(100 \mathrm{~kg} / \mathrm{ha})$ \\
\hline
\end{tabular}

FP: Fumiers de Poule ; BP : Burkina Phosphate

\section{RESULTATS \\ Production brute du riz pluvial strict}

Aucun investissement n'a été entrepris, nécessitant le calcul des amortissements ; alors, les coûts variables et les coûts fixes sont nettement les mêmes, et correspondent aux coûts de production. En faisant l'analyse suivant les pratiques de fertilisation, on constate que le traitement Compost+Urée, Compost+BP+Urée, BP+Urée et FP, ont des coûts de production bas (Tableau 3). Par contre les coûts de production les plus élevés ont été enregistrés par les traitements $\mathrm{FP}+\mathrm{BP}+$ Urée, $\mathrm{FP}+\mathrm{BP}, \mathrm{FP}+$ Urée, NPK+Urée et Compost+BP. Quant au produit brut en valeur, il a été plus faible sur les parcelles fertilisées aux FP, FP+BP, BP+Urée, Compost+BP et Compost+BP+Urée, qui sont statistiquement égaux. On note que les produits en valeurs sont statistiquement les mêmes pour les traitements NPK+Urée, Compost+Urée et FP+Urée. Le traitement $\mathrm{FP}+\mathrm{BP}+$ Urée enregistre significativement les produits en valeurs les plus élevées. A partir des résultats sur les coûts de production, on constate que la production d'un hectare de riz pluvial strict dans la zone d'étude nécessite un investissement non négligeable qui varie entre $90000 \mathrm{FCFA} / \mathrm{ha}$ et $230000 \mathrm{FCFA} / \mathrm{ha}$. Par rapport au témoin absolu, les traitements FP+Urée et $\mathrm{FP}+\mathrm{BP}+$ Urée entrainent une augmentation la valeur de la production brute de $134,02 \%$ et $153,9 \%$ respectivement.

\section{Indicateurs de performance économique et financière}

Les résultats relatifs à la Marge Nette (MN), à la Productivité Moyenne du Travail (PML), du Taux de Rentabilité Interne (TRI), du ratio Bénéfice-Coûts (RBC) sont consignés dans le Tableau 4. Il ressort des résultats d'analyse que les traitements Compost+Urée, $\mathrm{FP}+$ Urée et $\mathrm{FP}+\mathrm{BP}+$ Urée présentaient significativement la meilleure performance du point de vue de la marge nette, la productivité moyenne du travail du taux interne de rentabilité et du ratio bénéfice-coût.

Les marges nettes calculées révèlent que les coûts variables et les coûts fixes sont largement bien compensés par les produits bruts obtenus précédemment. Ainsi, toutes les marges nettes sont positives. De là, l'activité de production rizicole est économiquement rentable. Cependant, il y'a des disparités selon les options de fertilisation. Les marges nettes ont varié de $63428,147 \pm 91002,05$ FCFA/ha pour le traitement Compost+BP à $190439,933 \pm 70820,48$ FCFA/ha pour le traitement FP+Urée. Les traitements FP+Urée, $\mathrm{FP}+\mathrm{BP}+$ Urée, Compost+Urée et NPK+Urée qui ont le produit brut le plus élevé ont logiquement les marges nettes les plus élevées. Toutefois, l'ensemble des traitements ont des Marges Nettes (MN), des Productivité Moyenne du Travail (PML), des Taux de 
Rentabilité Interne (TRI), des Bénéfices sur Coût $(B / C)$ qui ne sont pas en moyenne significativement différentes. Ainsi, la productivité moyenne du travail varie de $21142,716 \pm 30334,02$ FCFA/hj pour le traitement Compost+BP à 63479,978 \pm 23606,83 FCFA/hj pour le traitement FP+Urée. Comparativement au témoin absolu, le traitement FP+Urée entraine une augmentation de PML de 170,4\%. En ce concerne le TRI, ce sont les traitements Compost+Urée et FP+Urée qui donnent significativement les taux de rentabilité interne les plus élevés suivi des traitements FP, $\mathrm{FP}+\mathrm{BP}, \quad \mathrm{FP}+\mathrm{BP}+$ Urée, $\mathrm{NPK}+$ Urée et Compost+BP+Urée. Par rapport au témoin absolu, les traitements Compost+Urée et $\mathrm{FP}+$ Urée entrainent des augmentations du TRI respectivement de 37 et $31,6 \%$. Les plus faibles taux de rentabilité interne ont été enregistrés par les traitements Compost+BP et BP+Urée qui sont statiquement égaux. L'analyse a montré que le ratio bénéfice-coût oscille entre $1,362 \pm 0,52$ et 2,071 $\pm 0,36$. En effet, le ratio bénéfice-coût est supérieur à la valeur 1 pour tous les traitements. Comparativement au traitement NPK+Urée, le traitement FP+Urée entraine une augmentation du ratio bénéfice- coût de 8,33\%. Par rapport au traitement témoin, le traitement FP entraine une augmentation du ratio bénéfice-coût de 5,5\%. Il ressort que les Ratio bénéfice-coût $(\mathrm{RBC})$ des traitements NPK+Urée, FP+BP+Urée et Compost+BP+Urée sont statistiquement égaux avec une moyenne de 1,65 $\pm 0,42$. Les traitements $\mathrm{BP}+$ Urée et Compost+BP sont statiquement les plus bas avec une moyenne de $1,417 \pm 0,45$.

\section{Corrélation entre indicateurs de performances et rendement paddy}

La matrice de corrélation est présentée dans le tableau 5. Les résultats indiquent des corrélations positive et significatives entre le rendement paddy et la marge nette d'une part et d'autre part entre la Productivité Moyenne du travail et le rendement paddy $(r=0,5)$. Une corrélation positive mais non significative $(r=0,25)$ existe entre le rendement paddy et le RBC et la corrélation $(r=0,25)$ entre le rendement paddy et le TRI positive mais non significative également. L'analyse statistique révèle que des corrélations positives et significatives $(r=1)$ existent entre le TRI et le RBC d'une part, et d'autre part entre le PML et le $\operatorname{RBC}(r=0,93)$.

Tableau 3 : Valeur de la production brute (PBV).

\begin{tabular}{lc}
\hline Traitements & PBV (FCFA/ha) \\
\hline Témoins absolu & $160427,71 *$ \\
\hline NPK+Urée & $355869,95 *$ \\
\hline BP+Urée & $228195,86^{*}$ \\
\hline FP & $291881,725^{*}$ \\
\hline FP+Urée & $375439,93 *$ \\
\hline FP+BP & $299588,04 *$ \\
\hline FP+BP+Urée & $407332,86 *$ \\
\hline Compost+Urée & $331291,073 *$ \\
\hline Compost+BP & $238428,14 *$ \\
\hline Compost+BP+Urée & $296234,89 *$ \\
\hline F pr. & $\mathbf{0 , 0 0 2}$ \\
\hline Signification & $*$ \\
\hline PBV : produit brut en valeur $; \mathbf{F P}:$ Fumiers de Poule $;$ BP : Burkina Phosphate $;$
\end{tabular}


Tableau 4 : Indicateurs de performances économiques selon les traitements.

\begin{tabular}{|c|c|c|c|c|}
\hline \multirow{2}{*}{ Traitements } & \multicolumn{4}{|c|}{ Indicateurs } \\
\hline & MN (FCFA/ha) & PML (FCFA/hj) & TRI & $\mathbf{R B C}$ \\
\hline Témoin absolu & $70427,717 \pm 29622,85^{*}$ & $23475,906 \pm 9874,28 *$ & $0,782 \pm 0,33^{*}$ & $1,782 \pm 0,33 *$ \\
\hline NPK+Urée & $165869,952 \pm 111441,65^{*}$ & $55289,984 \pm 37147,22 *$ & $0,873 \pm 0,59 *$ & $1,873 \pm 0,59 *$ \\
\hline BP+Urée & $73195,865 \pm 58785,71^{*}$ & $24398,622 \pm 19595,24 *$ & $0,472 \pm 0,38^{*}$ & $1,472 \pm 0,38^{*}$ \\
\hline FP & $136881,726 \pm 62243,36^{*}$ & $45627,242 \pm 20747,79^{* c}$ & $0,883 \pm 0,40^{*}$ & $1,883 \pm 0,4^{*}$ \\
\hline FP+Urée & $190439,933 \pm 70820,48^{*}$ & $63479,978 \pm 23606,83^{*}$ & $1,029 \pm 0,38^{*}$ & $2,029 \pm 0,38^{*}$ \\
\hline $\mathbf{F P}+\mathbf{B P}$ & $99588,045 \pm 48162,78^{*}$ & $33196,015 \pm 16054,26^{*}$ & $0,498 \pm 0,24^{*}$ & $1,498 \pm 0,24 *$ \\
\hline FP+BP+Urée & $177332,861 \pm 91980,96^{*}$ & $59110,954 \pm 30660,32 *$ & $0,771 \pm 0,4^{*}$ & $1,771 \pm 0,4^{*}$ \\
\hline Compost+Urée & $171291,073 \pm 5839 *$ & $57097,024 \pm 19463,33^{*}$ & $1,071 \pm 0,36^{*}$ & $2,071 \pm 0,36^{*}$ \\
\hline Compost+BP & $63428,147 \pm 91002,05 *$ & $21142,716 \pm 30334,02 *$ & $0,362 \pm 0,52 *$ & $1,362 \pm 0,52 *$ \\
\hline Compost+BP+Urée & $116234,896 \pm 87663,73 *$ & $38744,965 \pm 29221,24^{*}$ & $0,659 \pm 0,51^{*}$ & $1,659 \pm 0,51 *$ \\
\hline$F p r$. & 0,137 & 0,137 & 0,288 & 0,288 \\
\hline
\end{tabular}

FP : Fumier de Poule ; BP : Burkina Phosphate ; *significatif à 5\%

MN : Marge Nette ; PML : Productivité Moyenne du Travail ; TRI : Taux de Rentabilité Interne ; RBC : Ratio Bénéfice/Coût.

Tableau 5 : Matrice de corrélation.

\begin{tabular}{llllll}
\hline & MN & PML & TRI & RBC & Rdt \\
\hline MN & 1 & & & & \\
PML & 1 & 1 & & & \\
TRI & $0,93^{* *}$ & $0,93^{* *}$ & 1 & & \\
RBC & $0,93^{* *}$ & $0,93^{* *}$ & $1 * *$ & 1 & \\
Rdt & $0,5^{* *}$ & $0,5^{* *}$ & 0,25 & 0,25 & 1 \\
\hline
\end{tabular}

MN : Marge Nette; PML: Productivité Moyenne du Travail; TRI : Taux de Rentabilité Interne; RBC : Ratio Bénéfice/Coût ; Rdt : Rendement de riz paddy

NB : ** La corrélation est significative au niveau 0,01 (bilatéral). 


\section{DISCUSSION}

\section{Rentabilité économique}

Plusieurs travaux de recherche ont montré que l'utilisation des engrais inorganiques et autres amendements, sont considérés comme essentiel à l'accroissement de la production et à l'amélioration de la productivité (Akpo et al., 2016). Il faut noter que l'utilisation des engrais minéraux à un effet négatif et significatif sur la rentabilité économique des producteurs. Pour Biaou et al. (2016), le coût d'acquisition élevé des fumures minérales entraine une augmentation du coût total de production. Ce qui explique la marge nette faible enregistrée, et ne permet pas au producteur de rentabiliser sa production. L'apport de la fumure minérale a eu un effet négatif et significatif sur la marge nette. Cela confirme les résultats issus de l'étude de Labiyi et al. (2018) sur les pratiques culturales de gestion de la fertilité des sols et performance économique des producteurs de maïs au NordBénin. Les marges nettes pour l'ensemble des traitements ont été positives indiquant que les traitements sont économiquement rentables. Toutefois, elle est plus rentable avec les biodéchets associés aux engrais minéraux (FP+BP+Urée, FP+Urée et Compost+Urée) qu'avec les engrais minéraux et le Burkina Phosphate seul. Nos résultats confirment ceux de Mahaman (2001) et Adjiba (2016), qui ont montré que le fait que le producteur utilise l'engrais minéral, améliore sa performance technique mais ne lui permet pas de rentabiliser son exploitation. Ainsi donc, les crédits contractés pour l'achat des engrais seront difficilement remboursables. On peut conclure à cet effet, que le riziculteur sera insolvable s'il avait recours à une institution financière pour contracter un prêt destiné au financement des activités de son exploitation. Cette situation pourrait être une contrainte dans l'adoption de Burkina phosphate et des engrais minéraux en riziculture pluviale stricte. Selon Kohio et al. (2017), les problèmes matériels et financiers sont les principales contraintes d'adoption des bonnes pratiques de gestion durable des terres dans les zones soudaniennes du Burkina Faso.
Dans le même ordre d'idée, Yabi et al. (2016) ont montré que l'utilisation de l'engrais minéral améliore la performance technique du producteur, mais ne lui permet pas de rentabiliser son exploitation. En effet, si les engrais minéraux sont facilement solubles, leur bonne assimilation par les plantes dépend des bonnes propriétés physique, chimique et biologique des sols (Schoebitz et al., 2016). Une fertilisation adéquate des engrais minéraux sous culture est nécessaire pour améliorer leurs rendements. Mais les charges liées à sa pratique rendent la production non rentable. C'est pourquoi, son association avec la fertilisation organique permet d'augmenter les rendements du riz paddy.

Les marges nettes positives élevées observées pour les traitements $\mathrm{FP}+\mathrm{BP}+$ Urée, FP+Urée et Compost+Urée, s'expliquent par le fait que ces options ne nécessitent pas beaucoup d'engrais chimiques, et sont majoritairement constitués de produits locaux et accessibles à moindre coût. Ceci indique que la production de riz pluvial strict sous ces trois options de fertilisation (Compost+Urée, $\mathrm{FP}+$ Urée et $\mathrm{FP}+\mathrm{BP}+$ Urée), est une activité économiquement rentable. Les marges nettes obtenues restent supérieures à celle de la riziculture pluviale estimée à 142450 FCFA/ha FCFA/ha en moyenne au Nord-Est du Bénin (Yabi et al., 2012), et à celle de la Micro-dose + Bande enherbée + compost sous culture de sorgho, maïs et du mil estimée à 377 926,76 FCFA/ha dans les provinces du Zondoma et du Kouritenga au Burkina Faso (Sigue et al., 2019). L'utilisation des biodéchets, seuls ou associés à l'urée, améliore la productivité moyenne de travail pour les riziculteurs. Il serait préférable pour un riziculteur, de travailler dans sa propre exploitation plutôt que d'aller vendre sa force de travail sur le marché sous utilisation de biodéchet seule ou en combinaison avec l'urée et $\mathrm{BP}$, car cela permettrait de rémunérer le travail fourni par la main d'œuvre supplémentaire. Les biodéchets, seuls ou associés au BP et à l'urée, permettent donc aux producteurs d'obtenir de meilleurs rendements. Les productivités moyennes du 
travail des traitements $\mathrm{FP}+$ Urée, $\mathrm{FP}+\mathrm{BP}+$ Urée et Compost+urée sont supérieures à la valeur rapportée par Yabi et al. (2012) pour la riziculture au Nord-Est du Bénin, estimée à 4 848,96 FCFA/homme.jour en moyenne, et à celle de la technique de Micro-Dose + Zaï + Fumier estimée à 8 916,69 FCFA/homme.jr obtenue au Burkina Faso sous culture de sorgho, maïs et du mil (Sigue et al., 2019). De plus, le taux de rentabilité interne est élevé et significatif, pour les traitements FP, FP+Urée, $\mathrm{FP}+\mathrm{BP}+$ Urée, Compost +Urée et NPK+Urée. Ce taux est significativement supérieur au taux d'intérêt (24\%) pratiqué par les institutions de micro-finance de la zone d'étude. Ces taux obtenus sont supérieurs aux taux de rentabilité de la riziculture estimé à 0,01 par Yabi et al. (2012) dans la commune de Malanville au Bénin, et par Labiyi et al. (2019) pour l'utilisation de Cordons pierreux + Compost $(0,17)$ pour la culture du mil dans la commune de Bani au Burkina Faso. Ainsi, si les producteurs de riz pluvial strict devraient prendre un crédit auprès des institutions financières, il y'a certitude du remboursement, mais aussi de la viabilité de l'activité.

\section{Rentabilité financière}

Les résultats obtenus avec le ratio Bénefice-Coût confortent ceux obtenus précédemment par rapport aux marges nettes et à la valorisation du capital. On constate que le Ratio Bénéfice-Coût (RBC) est supérieur à 1 pour l'ensemble des traitements. Alors 1 franc investi génère plus de 1 FCFA comme bénéfice, et l'activité de production de riz pluvial sous les fertilisants est financièrement rentable. Par contre, les options de fertilisation prises séparément, on gagne avec les traitements FP+Urée et Compost+urée plus qu'avec les traitements NPK+Urée et $\mathrm{FP}+\mathrm{BP}+$ Urée si on investit un 1 franc dans la production de riz pluvial strict (en moyenne 2,05 franc contre 1,82 franc respectivement). Comme explication de cette situation, l'accès aux biodéchets qui est plus facile à moindre coût semble être un facteur discriminant de la rentabilité financière des fertilisants organiques et inorganiques. La rentabilité financière de ces fertilisants à base de compost et fumiers de poules peut s'expliquer par le fait que les amendements, associés à la fumure minérale, ont permis d'améliorer les rendements du riz paddy par rapport par à la fumure minérale seule.

Les Ratios Bénéfice sur Coût obtenus indiquent par ailleurs que la production du riz pluvial strict sous les traitements Compost+urée, est financièrement plus rentable que la production du sorgho Microdose avec Bandes enherbées avec Compost, qui présente un RBC estimé à 2,6 en moyenne au Kouritenga et au Zandoma au Burkina Faso (Sigue et al., 2019). De plus, les ratios $\mathrm{B} / \mathrm{C}$ obtenus avec les traitements sont supérieurs à ceux obtenus par Labiyi et al. (2019) sous pratique de Cordons pierreux+Compost (1,17). De plus, les Ratios Bénéfice sur Coût obtenus avec les traitements sont similaires à ceux évoqués par Sanou et al. (2016) dans un système de riziculture intensive dans les périmètres rizicoles irrigués de Karfiguéla et de la vallée du Kou au Burkina Faso. L'obtention de meilleurs rapport Bénefice-Coût avec les traitements FP, $\mathrm{FP}+$ Urée et $\mathrm{FP}+\mathrm{BP}+$ Urée pourraient s'expliquer par le fait que les biodéchets (Fumier de Poule) utilisés seuls ou combinés, pourraient améliorer le rendement du riz pluvial strict (Sanon et al., 2021). La performance financière de ces trois options de fertilisation pourrait s'expliquer par le fait que les biodéchets utilisés seul ou combinés à l'urée, permettent d'améliorer la disponibilité des éléments nutritifs des sols, et conséquemment le rendement du riz pluvial strict. Ceci serait dû à la grande quantité d'azote libérée par ces traitements, et qui est profitable au début du tallage et à la montaison. Selon Henuk et al. (2003), les déchets fourragers contenus dans le fumier peuvent être utilisés de manière économique comme source d'engrais. En général, les éléments nutritifs de base dans le fumier de poule (N, P, K) sont les mêmes comme dans les engrais commerciaux, et sont tout aussi efficaces pour favoriser la 
croissance des plantes. Par conséquent, le fumier de poule doit être considérés comme une ressource d'engrais et géré dans la culture d'une manière similaire aux engrais commerciaux (Fulhage, 1992).

Pour l'ensemble de traitements, la rentabilité financière a été obtenue avec la dose de FP et ou Compost combinée à la faible dose de l'Urée. Les fortes doses de fertilisants exigent une augmentation du coût de production, entrainant une réduction du profit. Et même, lorsque les fortes doses des fertilisants sont utilisées, l'augmentation de rendement qu'elles entrainent ne fait que compenser les dépenses engagées pour leur achat. Les études similaires faites à Lubumbashi, RD Congo par Useni et al. (2012) par l'utilisation de 1,75 tonne FP, $150 \mathrm{~kg}$ NPK et $100 \mathrm{~kg}$ urée. Par ailleurs au Cameroun Kaho et al. (2011) ont également montré que le traitement aux fertilisants organiques seuls (5t.ha ${ }^{-1}$ de Feuilles de Tithonia diversifolia) procurait les marges bénéficiaires les plus élevées que les traitements avec engrais minéraux seuls ou combinés aux fertilisants organiques.

\section{Relation entre indicateurs de rentabilité et rendement de riz paddy}

La corrélation entre le rendement de riz paddy obtenu et le bénéfice sur coûts utilisés, est positive, cependant non significative. Cela signifie qu'une augmentation indéfinie des fertilisants organiques et inorganiques, à un effet sur la production, en revanche les coûts variables ne pourront pas être compensés par les produits bruts obtenus. Cela confirme les résultats issus de l'étude de Labiyi et al. (2018), portant sur les pratiques culturales de gestion de la fertilité des sols et performance économique des producteurs de maïs au Nord du Bénin. Toutefois, les traitements, FP+Urée $\mathrm{FP}+\mathrm{BP}+$ Urée et Compost+Urée, donnent les meilleurs indicateurs de performance économique et financière. Ces traitements sont alors économiquement rentables. L'association du Compost, BP, FP et/ou à l'urée, a favorisé non seulement l'assimilation des engrais minéraux, mais aussi a libéré les éléments nutritifs contenus dans les biodechets, générant la bonne performance de ces traitements. Selon Useni et al. (2012), les fortes concentrations en éléments nutritifs des biodéchets et de leur impact positif sur les propriétés chimiques, expliqueraient cette performance sur l'augmentation des rendements paddy du riz pluvial. Ainsi, la combinaison biodéchet et fumure minérale, et l'application seule de biodéchet, permettent de réduire les charges liées à l'achat d'engrais pour la fertilisation du sol, d'où leur influence sur la marge nette, la productivité moyenne du travail. Ceci s'est traduit par une forte relation entre rendement paddy et ces indicateurs.

\section{Conclusion}

L'objectif de cette étude était d'évaluer les performances économiques et financières des options de fertilisation en vue de l'amélioration de la productivité des exploitations de riz pluvial strict. Les résultats montrent que les traitements NPK+Urée, $\mathrm{FP}+\mathrm{BP}+$ Urée et Compost+BP+Urée, engendrent des produits bruts en valeur à l'hectare plus élevés. Toutefois, en tenant compte des coûts totaux, les engrais minéraux diminuent la performance économique et financière de la production de riz pluvial strict. Par contre, les traitements FP+Urée, $\mathrm{FP}+\mathrm{BP}+$ Urée et Compost+Urée améliorent la rentabilité économique et financière de la production du riz pluvial strict. L'utilisation de biodéchets, seuls ou combinés avec les fertilisants inorganiques, améliore la performance économique et financière de la fertilisation en riziculture pluviale stricte. En effet, le coût d'acquisition élevé des fumures minérales et du Burkina Phosphate, fait augmenter les coûts totaux de production de riz pluvial strict. Il y a donc une impérieuse nécessité d'accompagner des riziculteurs par la subvention du Burkina phosphate, et de faire la promotion de l'intégration agriculture/élevage par la prise en compte suffisante du volet élevage de Poules pour une meilleure 
valorisation des ressources locales de fertilisation.

\section{CONFLITS D'INTERETS}

Les auteurs déclarent qu'il n'y a aucun conflit d'intérêts.

\section{CONTRIBUTIONS DES AUTEURS}

Cet article est l'œuvre d'un auteur principal (AS) et des co-auteurs (APKG et HS). AS est l'auteur qui a rédigé le protocole de recherche et conduit les travaux sur le terrain avec l'appui de CB, WS, et SF, conçu la base de données et analysé les données. C'est lui, AS qui a proposé le premier draft de l'article, géré le processus de soumission et a suivi les recommandations des lecteurs sous la supervision du co-auteur APKG et HBN. Par ailleurs, HS a contribué à la validation du protocole de recherche à l'élaboration de la partie théorique de la première version jusqu'à celle-ci. Il a participé également à l'analyse des données. $\mathrm{KC}$ a participé à la rédaction du protocole.

\section{REFERENCES}

Adebiyi KD, Maiga-Yaleu S, Issaka K, Ayena MEt Yabi JA. 2019. Déterminants de l'adoption des bonnes pratiques de gestion durable des terres dans un contexte de changement climatique au Nord Bénin : cas de la fumure organique. International Journal of Biological and Chemical Sciences, 13(2): 998-1010. DOI:

https://dx.doi.org/10.4314/ijbcs.v13i2.34

Adjiba C., 2016. Analyse des performances techniques et économiques de quelques pratiques de gestion de la fertilité des sols dans les systèmes de culture à base de maïs au Nord Bénin. Mémoire de Master Professionnel. Faculté d'Agronomie/Université de Parakou, 115 p.

Akanza PK, Yoro G. 2003. Effets synergiques des engrais minéraux et de la fumure de volailles dans l'amélioration de la fertilité d'un sol ferrallitique de l'ouest de la Côte d'Ivoire. Agronomie Africaine, 15 (3): 135-144. DOI: 10.4314/aga.v15i3.1631

Akpo MA, Saïdou A, Yabi I, Balogoun I, Bio Bigou BL. 2016. Evaluation de la performance des pratiques de gestion de la fertilité des sols dans le bassin de la Rivière Okpara au Benin. European Scientific Journal, 12 (33): 370-390. DOI:

http://dx.doi.org/10.19044/esj.2016.v12n 33p370

Biaou D, Yabi1 JA, Yegbemey RN, Biaou G. 2016. Performances technique et économique des pratiques culturales de gestion et de conservation de la fertilité des sols en production maraîchère dans la commune de Malanville, Nord Bénin. International Journal of Innovation and Scientific Research, 21 (1): 201-211. http://citeseerx.ist.psu.edu/viewdoc/dow nload?doi=10.1.1.678.2007\&rep=rep1\&t ype $=$ pdf

DDEA (Stratégie Nationale de Développement de l'Entrepreneuriat Agricole à l'horizon 2025) 2012. Rapport Final. 57 p.

Eureka 2005. Revue trimestrielle du Centre National de la Recherche Scientifique et Technologique (CNRST), Burkina Faso, Partenariat ADRAO - INERA, plus de dix ans aux services du développement rizicole. $74 \mathrm{p}$.

INERA (Institut National de l'Environnement et des Recherches Environnementales) 2016. Programme Riz et Riziculture, Fiche technique de la variété de riz FKR59 (WAB99-84),

INSD (Institut National de la Statistique et de la Démographie), 2020. Résultats préliminaires. Cinquième Recensement Général de la Population. 55 p.

Kone K, Sylvester O, Diatta E, Somado S, Valere K, Sahrawat KL. 2011. Response of interspecific and sativa upland rices to Mali phosphate rock and soluble phosphate fertilizer. Archives of 
Agronomy and Soil Science, 57 (4): 421434.

DOI:

https://doi.org/10.1080/03650340903563 382

Kaho F, Yemefack M, Feujio-Tegwefouet P, Tchanthaouang JC, 2011. Effet combiné de feuilles de Tithonia diversifolia et des engrais inorganiques sur le rendement $\mathrm{du}$ maïs et les propriétés d'un sol ferralitique au centre du Cameroun. Tropicultura, 29 (1): $39-45$

Kissou R, Traoré E, Gnankambary Z, Nacro HB, Sédogo PM. 2014. Connaissance endogène de la classification et de la fertilité des sols en zone SudSoudanienne du Burkina Faso. VertigO, revue électronique en sciences de l'environnement, 14 (1): 1-17. https://id.erudit.org/iderudit/1027966ar

Kohio EN, Toure AG, Sedogo MP, Karimou JM, Ambouta KJM. 2017. Contraintes à l'adoption des bonnes pratiques de Gestion Durable des Terres dans les zones soudaniennes et soudano-sahéliennes du Burkina Faso. International Journal of Biological and Chemical Science, 11 (6): 2982-2989.

DOI: https://dx.doi.org/10.4314/ijbcs.v11i6.34

Lacharme M. 2001. Le plant de riz : Données morphologiques et cycle de la plante, Mémento Technique de Riziculture. 22 p. Labiyi AI, Yegbemey RN, Olodo VD, Yabi JA. 2018. Pratiques culturales de gestion de la fertilité des sols et performance économique des producteurs de maïs au Nord Bénin. Annales de l'Université de Parakou, Série Sciences Naturelles et Agronomie, $8 \quad$ (2): 115-124. https://www.researchgate.net/publication /334121902

Labiyi IA, Sigué H, Ouattara D, Traoré OM, Koura D. 2019. Effet des pratiques innovantes endogènes de gestion durable des terres sur la performance technicoéconomique du réseau de producteurs dans la commune de Mani au Burkina
Faso. Afrique Science, 15 (1) : 432 - 447. http://www.afriquescience.net

Mahaman I. 2001. Evolution à long terme de la fertilité de sol dans la région de Maradi. Rapport d'étude, Drylands Research Crewkerne, Somerset, Royaume-Uni, 44p.

MAAH (Ministère de l'Agriculture et des Aménagements Hydro-Agricoles du Burkina Faso). 2018. Annuaire des Statistiques Agricoles, 2017. 118 p.

MAAH (Ministère de l'Agriculture et des Aménagements Hydro-Agricoles du Burkina Faso). 2020. Annuaire des Statistiques Agricoles 2019. 408 p.

MCIA (Ministère du Commerce, de Burkina Faso l'Industrie et de l'Artisanat). 2020. Balance commerciale et commerce extérieur du Burkina Faso. 60 p.

Onaga G, Asea G, Lamo J, Kikafunda J, Bigirwa G. 2012. Comparison of Response to Nitrogen between Upland NERICAs and ITA (Oryza sativa) Rice Varieties. Journal of Agricultural Science, 6 (4): 197-205. DO I: 10.5539/jas.v4n6p197

Sanon A, Gomgnimbou APK, Coulibaly K, Nacro HB. 2021. Effets de biodéchets et de fertilisants inorganiques sur la production du riz pluvial strict en zone sud-soudanienne du Burkina Faso. Afrique Science, 18 (1) : 230 - 241. http://www.afriquescience.net

Sanou AG, Dembele KD, Ouedraogo I, Dakouo D. 2016. Problématique de mise en œuvre du système de riziculture intensif dans les périmètres rizicoles irrigués de Karfiguéla et de la vallée du Kou au Burkina Faso. International Journal of Biological and Chemical Sciences, 10 (6): 2693-2709. DOI : http://dx.doi.org/10.4314/ijbcs.v10i6.23

Schoebitz M, Vidal G. 2016. Microbial consortium and pig slurry to improve chemical properties of degraded soil and nutrient plant uptake. Journal of Soil 
Science and Plant Nutrition, 16 (1): 226236. DOI:

http://dx.doi.org/10.4067/S071895162016005000018

Sigue H, Labiyi IA, Issaka K, Yabi JA, Biaou G. 2019. Effet Des Composantes de la Technologie microdose sur la Performance Économique et financière des Exploitations Agricoles du Kouritenga et du Zondoma Au Burkina Faso. African Crop Science Journal, 27 (3) : 331-349. DOI:10.4314/acsj.v27i3.2

SNDR. 2020. (Deuxième Génération de la Stratégie Nationale de Développement de la Riziculture 2021-2030). Ministère de l'Agriculture et des Aménagements Hydro-Agricoles. Burkina Faso, 120 p.

Steel RGD, Torrie JH, Dickey DA. 1997. Principles and Procedures of Statistics. A Biometrical Approach. McGraw Hill Book Co.: New York, USA; 400-428.

Useni SY, Baboy LL, Nyembo KL, Mpundu MM. 2012. Effets des apports combinés de biodéchets et de fertilisants inorganiques sur le rendement de trois variétés de Zea mays L. cultivées dans la région de Lubumbashi. Journal of Applied Biosciences, 54: 3935-3943.
Yabi JA, Bachabi FX, Labiyi IA, Ode CA, Ayena RL. 2016. Déterminants socioéconomiques de l'adoption des pratiques culturales de gestion de la fertilité des sols utilisées dans la commune de Ouaké au Nord-Ouest du Bénin. International Journal of Biological and Chemical Sciences, 10 (2): 779-792. DOI: 10.4314/IJBCS.V10I2.27

Yabi AJ, Paraïso A, Yegbemey R, Chanou P. 2012. Rentabilité Economique des Systèmes Rizicoles de la Commune de Malanville au Bénin. Bulletin de la Recherche Agronomique du Bénin (BRAB), $\mathrm{N}^{\circ}$ spécial, Productions Végétales \& Animales et Economie \& Sociologie Rurales : 1-12. https://www.researchgate.net/publication 1234143780

Yegbemey RN, Yabi JA, Aihounton GB, Paraiso A. 2014. Modélisation simultanée de la perception et de l'adaptation au changement climatique : cas des producteurs de maïs du Nord Bénin (Afrique de l'Ouest). Cahier d'Agriculture, 23 (3): 177-187. DOI : https://doi.org/10.1684/agr.2014.0697 